\title{
AVALIAÇÃO QUÍMICA DO INHAME (Colocasia esculenta L. Schott) CULTIVADO EM SOLO ALAGADIÇO NA REGIÃO PANTANEIRA DE MATO GROSSO DO SUL
}

\author{
MANOEL MENDES RAMOS FILHO * \\ MARIA ISABEL LIMA RAMOS * \\ PRISCILA AIKO HIANE *
}

\begin{abstract}
Amostras de rizomas laterais de inhame (Colocasia esculenta L. Schott) variedades cem/um, branco, chinês, japonês e macaquinho, cultivadas experimentalmente na Base de Estudos do Pantanal da UFMS foram analisadas com o objetivo de estabelecer e avaliar dados da composição centesimal e teores de minerais nutrientes. O teor de umidade variou de 62,78 (chinês) a 78,92\% p/p (macaquinho); as variedades chinês e japonês apresentaram teores de proteínas equivalentes ( 2,86 e $3,02 \% \mathrm{p} / \mathrm{p}$, respectivamente) e superiores às demais. Todos os inhames mostraram-se pobres em lipídios $(0,21$ a $0,23 \% \mathrm{p} / \mathrm{p})$. Quanto à fração fibra, os valores diferenciaram-se entre os cultivares, oscilando de 1,31 a $2,93 \%$ p/p. A variedade japonês apresentou teor de resíduo mineral fixo $(1,15 \% \mathrm{p} / \mathrm{p})$ superior ao das outras variedades $(0,90 \% \mathrm{p} / \mathrm{p})$, constituindo-se na melhor fonte de minerais macro nutrientes $(218,35 \mathrm{mg} /$ $100 \mathrm{~g}$ ), enquanto que o maior teor de minerais micro nutrientes coube à variedade macaquinho (24,13 ppm). A concentração de amido existente nos cultivares analisados variou de 14,46 a $30,30 \%$ $\mathrm{p} / \mathrm{p}$, sendo este último valor encontrado na variedade chinês. A avaliação dos resultados das determinações analíticas realizadas evidenciou que a variedade chinês é a melhor fonte de energia e a matéria-prima de melhor aproveitamento industrial.
\end{abstract}

\footnotetext{
* Professores do Departamento de Tecnologia de Alimentos e Saúde Pública (DTA), Centro de Ciências Biológicas e da Saúde, Universidade Federal do Mato Grosso do Sul (UFMS), Campo Grande, MS.
} 
Várias espécies de plantas tropicais pertencentes à família Araceae são conhecidas no Brasil pelo nome de inhame, muitas vezes confundido com o cará, espécie esta do gênero Dioscorea e família Dioscoreaceae $(4,5,15)$.

O inhame (Colocasia esculenta L. Schott), cujo caule subterrâneo apresenta um rizoma central circundado de 6 a 20 rizomas laterais, tem vários sinônimos estrangeiros, como "alcoalcas", "dasheen", "taro", "malangay", "tania", "tanier" $(4,13,15)$.

As aráceas, conhecidas na China há 100 anos a.C., constituem-se em uma das mais antigas plantas cultivadas, com inúmeras variedades disponíveis atualmente. Plantadas por povos das regiões tropicais e subtropicais, onde o cultivo de outra olerácea, como por exemplo a batata, é afetado pela alta temperatura local, são largamente consumidas no Egito, Filipinas, Hawai e algumas ilhas do Caribe e Pacífico, onde assumem considerável valor comercial $(8,17)$.

Economicamente o inhame é um alimento barato, apresentando elevado rendimento por unidade de área plantada. Características de rusticidade edafo-climáticas, resistência a pragas e doenças, alta produtividade e fácil conservação pós-colheita favorecem o seu cultivo em áreas inaproveitáveis pela agricultura tradicional, como solos alagados e pantanais $(8,19)$.

Sob o ponto de vista nutricional, o inhame é superior à batata inglesa, batata doce, mandioca, cará, e ao arroz. Seus rizomas são ricos em carboidratos, proteínas, vitaminas do complexo B e minerais. Embora de baixo conteúdo em lipídios, apresenta alto nível de ácidos graxos insaturados, além de antocianinas $(3,8,14,15,17)$.

O fator desfavorável do inhame, que pode ser eliminado através do cozimento, seria a presença de acridez, causadora de irritação da mucosa oral e da epiderme, a qual estaria associada a cristais de oxalato de cálcio liberados do interior de células especiais, denominadas idioblastos $(11,12)$.

O valor do inhame, como matéria-prima alimentícia, foi atribuído às características de fácil digestibilidade dos grânulos de amido, alto valor energético, alto conteúdo de vitamina $\mathrm{B}$, teores elevados de cálcio, fósforo e ferro, além da alcalinidade das cinzas e da não alergenicidade $(8,11,15,17)$.

Facilmente assimilado pelo organismo, o amido do inhame pode ser usado em alimentos infantis, alimentos hipoalérgicos e como sucedâneo de cereal em dietas destinadas às pessoas com doença celíaca $(3,6,11,13)$. 
Em países da Ásia e Pacífico, o inhame é usado como substituto do arroz. No Hawai seu consumo se dá principalmente na forma de um produto pastoso, obtido por fermentação, denominado "poi" $(3,11,13)$.

À semelhança de outras raízes, o inhame pode ser consumido assado, cozido ou frito em óleo, e quando transformado em farinha pode ser utilizado na produção de sopas, biscoitos, pães, bebidas e pudins $(5,11,13)$.

SIVIERO et al. (17) estudaram o processamento e a utilização da farinha de inhame em produtos de panificação, e demonstraram a viabilidade da substituição da farinha de trigo pela de inhame. Segundo os mesmos autores, os melhores resultados foram obtidos substituindo-se 15, 20 e $30 \%$ do trigo nas formulações para pão, biscoito e bolo, respectivamente.

MOY et al. (11) desenvolveram derivados extrusados com formato de grão de arroz, talharim e espaguete a partir de farinha de inhame, obtendo conforme os autores, produtos de boa qualidade sensorial, com menor tempo de cocção que os similares comerciais.

A necessidade e a importância do estudo e cultivo do inhame num país como o Brasil, onde a maioria da população carece de alimentos, bem como a caracterização química de produtos regionais para busca de dados sobre o valor nutricional de matérias-primas que representam potencialidades para o Estado de Mato Grosso do Sul, justificam o presente trabalho. Visando a obtenção de novas fontes alimentícias com viabilidade econômica avaliou-se o inhame, em bases regionais, quanto aos valores de nutrientes fornecedores de energia e de macro e micro elementos biorreguladores em diferentes cultivares.

\section{MATERIAL E MÉTODOS}

\subsection{PREPARO DAS AMOSTRAS}

Cerca de $2,0 \mathrm{~kg}$ de amostras de rizomas laterais dos cinco cultivares de inhame (Colocasia esculenta L. Schott) procedentes do cultivo experimental na Base de Estudos do Pantanal da UFMS, município de Miranda-MS, passaram por triagem e limpeza, descartando-se aqueles com sinais de deterioração. Posteriormente, foram pesados em balança semi-analítica Marte Mod. A 5000, descascados e fatiados mecanicamente em multiprocessador Walita Master Plus, equipado com disco descascador DB01. Alíquotas foram utilizadas para a determinação de umidade e o restante do material colocado nas bandejas e desidratado em estufa com circulação forçada de ar, em temperatura de $60^{\circ} \mathrm{C}$. As amostras desidratadas, trituradas em moinho de martelo Tecnal Mod. TE 090 e 
peneiradas em tamiz 60 tyler, ficaram acondicionadas em recipientes de vidro com tampa rosqueável durante a realização das análises.

\subsection{ANÁLISES}

\subsubsection{Umidade e substâncias voláteis}

Foram determinadas por método gravimétrico em estufa a $105^{\circ} \mathrm{C}$, conforme descrito pelo INSTITUTO ADOLFO LUTZ (9).

\subsubsection{Resíduo mineral fixo}

Calculado por gravimetria, após carbonização de amostras em chama direta e posterior incineração em mufla a $550^{\circ} \mathrm{C}$, de acordo com o preconizado pelo INSTITUTO ADOLFO LUTZ (9).

\subsubsection{Extrato etéreo}

Foi determinado gravimetricamente após a vaporização do éter de petróleo, empregado como solvente extrator em aparelho de Soxhlet, segundo procedimento adotado pelo INSTITUTO ADOLFO LUTZ (9).

\subsubsection{Proteínas}

Utilizou-se o método micro Kjeldahl para estimar o nitrogênio total, empregando-se o fator 6,25 para convertê-lo em proteína, de acordo com a ASSOCIATION OF OFFICIAL ANALYTICAL CHEMISTS (1).

\subsubsection{Fibra}

O conteúdo de fibra foi avaliado pelo método da fibra detergente neutro, modificado para amostras contendo amido e a fibra determinada através de solução detergente neutro, conforme descrito por MENDEZ et al. e VAN $\operatorname{SOEST}(10,18)$.

\subsubsection{Carboidratos}

Os açúcares redutores em glicose e os não redutores em sacarose obtidos por extração alcoólica, segundo o INSTITUTO ADOLFO LUTZ (9), foram quantificados por espectrofotometria com os reagentes de SomogyiNelson, conforme descrito por AUED et al. (2), em comprimento de onda de $500 \mathrm{~nm}$, em aparelho Spectronic 70 da Bausch \& Lomb. 
O conteúdo em amido foi determinado no resíduo da extração alcoólica, após hidrólise ácida à quente, pelo método Lane-Eynon, com o reativo de Fehling, segundo procedimento do INSTITUTO ADOLFO LUTZ (9).

\subsubsection{Minerais}

O teor de minerais foi avaliado no Laboratório de Nutrição Animal do Centro de Pesquisa de Gado de Corte da Embrapa/Campo Grande-MS, segundo metodologia descrita por SALINAS e GARCIA (16). Empregou-se espectrofotômetro de absorção atômica, da Perkim-Elmer Mod. 306, para determinar os teores dos minerais ferro, manganês, zinco, cobre, cálcio e magnésio; o fotômetro de chama da Micronal Mod. B 262 para os minerais sódio e potássio, e o espectrofotômetro-luz visível da Micronal Mod. B 382 para o mineral fósforo.

\subsubsection{Análise estatística}

Os resultados obtidos na determinação da composição centesimal foram submetidos à análise de variância (ANOVA) e as diferenças observadas analisadas pelo teste de Tukey, conforme descrito por GOMES (7).

\section{RESULTADOS E DISCUSSÃO}

\subsection{COMPOSIÇÃO CENTESIMAL}

Os resultados obtidos nas determinações da composição centesimal realizadas nos rizomas laterais de inhame cem/um, branco, chinês, japonês e macaquinho encontram-se na Tabela 1.

Dentre os valores de umidade obtidos nota-se variação de 62,78 a $78,92 \% \mathrm{p} / \mathrm{p}$ nos resultados. Diferenças significativas ao nível de $5 \%$ de probabilidade foram verificadas nos teores de umidade entre algumas variedades de inhame. Comparando-se os teores de umidade, entre as variedades cem/um e japonês, branco e japonês, observou-se não haver diferença significativa ao nível de $5 \%$ de probabilidade.

Quanto aos teores de proteína obtidos, as variedades chinês (2,86\% p/p) e japonês $(3,02 \% \mathrm{p} / \mathrm{p})$ apresentaram equivalência, da mesma forma que o cem/um $(2,56 \% \mathrm{p} / \mathrm{p})$ e o branco $(2,60 \% \mathrm{p} / \mathrm{p})$. O menor conteúdo protéico foi observado na variedade macaquinho $(2,19 \% \mathrm{p} / \mathrm{p})$. 

TABELA 1 - COMPOSIÇÃO CENTESIMAL DAS CINCO VARIEDADES DE INHAME ORIUNDAS DE SOLO ALAGADIÇO DA REGIÃO PANTANEIRA DE MATO GROSSO DO SUL, EXPRESSA EM $g / 100 \mathrm{~g}$ DE AMOSTRAS NO ESTADO FRESCO $\pm S^{*}$

\begin{tabular}{lccccc}
\hline DETERMINAÇÕES** & CEM/UM & BRANCO & CHINÊS & JAPONÊS & MACAQUINHO \\
\hline UMIDADE & 74,37 & 73,78 & 62,78 & 76,26 & 78,92 \\
PROTEÍNAS & $\pm 0,57 \mathrm{BC}$ & $\pm 1,02 \mathrm{C}$ & $\pm 1,47 \mathrm{D}$ & $\pm 1,35 \mathrm{~B}$ & $\pm 0,95 \mathrm{~A}$ \\
& 2,56 & 2,60 & 2,86 & 3,02 & 2,19 \\
LIPÍDIOS & $\pm 0,03 \mathrm{~B}$ & $\pm 0,07 \mathrm{~B}$ & $\pm 0,07 \mathrm{~A}$ & $\pm 0,14 \mathrm{~A}$ & $\pm 0,02 \mathrm{C}$ \\
& 0,13 & 0,15 & 0,23 & 0,12 & 0,12 \\
FIBRA & $\pm 0,01 \mathrm{BC}$ & $\pm 0,01 \mathrm{~B}$ & $\pm 0,05 \mathrm{~A}$ & $\pm 0,01 \mathrm{C}$ & $\pm 0,01 \mathrm{C}$ \\
& 1,47 & 1,80 & 2,93 & 1,31 & 1,70 \\
RESÍDUO MINERAL & $\pm 0,04 \mathrm{D}$ & $\pm 0,07 \mathrm{~B}$ & $\pm 0,22 \mathrm{~A}$ & $\pm 0,06 \mathrm{E}$ & $\pm 0,01 \mathrm{C}$ \\
FIXO & 0,90 & 0,90 & 0,90 & 1,15 & 0,91 \\
AÇÚCARES & $\pm 0,03 \mathrm{~B}$ & $\pm 0,01 \mathrm{~B}$ & $\pm 0,02 \mathrm{~B}$ & $\pm 0,01 \mathrm{~A}$ & $\pm 0,03 \mathrm{~B}$ \\
REDUTORES & 0,31 & 0,22 & 0,10 & 0,22 & 0,80 \\
AÇÚCARES NÃO & $\pm 0,03 \mathrm{~B}$ & $\pm 0,03 \mathrm{C}$ & $\pm 0,02 \mathrm{D}$ & $\pm 0,03 \mathrm{C}$ & $\pm 0,13 \mathrm{~A}$ \\
REDUTORES & 0,75 & 0,78 & 0,86 & 0,43 & 0,90 \\
AMIDO & $\pm 0,04 \mathrm{~B}$ & $\pm 0,05 \mathrm{~B}$ & $\pm 0,02 \mathrm{~A}$ & $\pm 0,12 \mathrm{C}$ & $\pm 0,04 \mathrm{~A}$ \\
V.C.T.*** & 18,42 & 19,80 & 30,30 & 18,38 & 14,46 \\
kcal/100 g & $\pm 0,64 \mathrm{C}$ & $\pm 0,22 \mathrm{~B}$ & $\pm 0,67 \mathrm{~A}$ & $\pm 0,21 \mathrm{C}$ & $\pm 040 \mathrm{D}$ \\
* & 85,05 & 90,95 & 134,71 & 86,68 & 67,68 \\
** & & & & \\
médias na mesma linha & seguidas pela mesma letra não diferem entre si pelo teste de Tukey,
\end{tabular}

Todas as variedades de inhame estudadas apresentaram baixíssimo conteúdo de lipídios (entre 0,12\% a 0,23\% p/p), evidenciando contribuição insignificante na composição química dos rizomas.

Notou-se considerável variação entre os valores obtidos para fibra, que diferem significativamente ao nível de $5 \%$ de probabilidade. A variedade chinês obteve o maior teor de fibra $(2,93 \% \mathrm{p} / \mathrm{p})$ e a japonês $(1,31 \% \mathrm{p} / \mathrm{p}) \mathrm{o}$ menor.

Comparando-se os valores de resíduo mineral fixo não foi verificada diferença significativa entre as variedades de inhame, exceto pela variedade japonês, que apresentou teor de cinzas superior $(1,15 \% \mathrm{p} / \mathrm{p})$ ao das demais $(0,90 \% \mathrm{p} / \mathrm{p})$. 
Os açúcares redutores e os não redutores contribuíram individualmente com parcelas inferiores a $1 \%$ na composição centesimal das cinco variedades. O inhame macaquinho apresentou os maiores valores para ambos os açúcares, ao passo que para a variedade "chinês" encontrou-se somente $0,1 \% \mathrm{p} / \mathrm{p}$ de açúcares redutores.

$\mathrm{Na}$ composição centesimal dos rizomas, o teor de amido como componente majoritário variou de 14,46 a $30,30 \%$ p/p. Verificou-se que o teor de amido apresentado pela variedade chinês $(30,30 \% \mathrm{p} / \mathrm{p})$ correspondeu a mais que o dobro do encontrado para a variedade macaquinho (14,06\% p/p). Mostrou-se superior ao das variedades cem/um $(18,42 \% \mathrm{p} / \mathrm{p})$ e japonês $(18,38 \% \mathrm{p} / \mathrm{p})$ em $65 \%$, além de conter $53 \%$ a mais de amido que a variedade branco.

Os resultados evidenciaram que a variedade chinês apresentou o maior valor energético $(134,71 \mathrm{kcal} / 100 \mathrm{~g})$, fornecendo de 48 a $99 \%$ a mais de calorias que as outras variedades estudadas.

\subsection{MINERAIS MACRO NUTRIENTES}

Os valores encontrados para os minerais cálcio, magnésio, fósforo e potássio, estão apresentados na Tabela 2.

Observa-se para a variedade japonês teor elevado de cálcio $(74,80 \mathrm{mg})$ em comparação às demais, entre as quais o macaquinho mostrou o menor valor (apenas 8,48 mg).

\section{TABELA 2 - TEORES DE MINERAIS MACRO NUTRIENTES DAS CINCO VARIEDADES DE INHAME ORIUNDAS DE SOLO ALAGADIÇO DA REGIÃO PANTANEIRA DE MATO GROSSO DO SUL, EXPRESSOS EM mg/100 g DE AMOSTRA NO ESTADO FRESCO}

\begin{tabular}{cccccc}
\hline Mineral & Cem/um & Branco & Chinês & Japonês & Macaquinho \\
\hline Cálcio & 12,60 & 26,90 & 23,00 & 74,80 & 8,48 \\
Magnésio & 22,70 & 26,90 & 46,00 & 24,40 & 19,00 \\
Fósforo & 62,20 & 73,70 & 87,00 & 59,80 & 42,80 \\
Potássio & 45,80 & 41,40 & 41,00 & 59,35 & 45,62 \\
Total & 143,30 & 168,90 & 197,00 & 218,35 & 115,90 \\
\hline
\end{tabular}


Para o mineral magnésio, a variedade chinês apresentou conteúdo 2,42 vezes maior em relação à macaquinho, cujo valor encontrado foi inferior também ao das variedades cem/um, branco e japonês.

Quanto ao teor do mineral fósforo predominou a variedade chinês, seguido pela branco com teor próximo daqueles obtidos para os rizomas cem/um e japonês. A variedade macaquinho, com o menor teor de fósforo, mostrouse a pior fonte de minerais macro nutrientes dentre as variedades analisadas.

O conteúdo do mineral potássio oscilou entre 41,50 a 59,35 mg, mostrando valores semelhantes entre as variedades, com exceção da japonês responsável pelo maior teor $(59,35 \mathrm{mg})$.

Considerando o conteúdo total de minerais macro nutrientes, as melhores fontes em ordem decrescente foram as variedades japonês, chinês e branco.

\subsection{MINERAIS MICRO NUTRIENTES}

Os resultados obtidos para os minerais sódio, ferro, manganês, zinco e cobre são apresentados pela Tabela 3.

\section{TABELA 3 - TEORES DE MINERAIS MICRO NUTRIENTES NAS CINCO VARIEDADES DE INHAME ORIUNDAS DE SOLO ALAGADIÇO DA REGIÃO PANTANEIRA DE MATO GROSSO DO SUL, EXPRESSOS EM PARTE POR MILHÃO (ppm) DE AMOSTRA NO ESTADO FRESCO}

\begin{tabular}{cccccc}
\hline Mineral & Cem/um & Branco & Chinês & Japonês & Macaquinho \\
\hline Sódio & 9,26 & 7,59 & 4,09 & 7,91 & 8,96 \\
Ferro & 5,41 & 3,87 & 4,16 & 4,21 & 5,18 \\
Manganês & 3,32 & 1,58 & 2,58 & 3,96 & 3,39 \\
Zinco & 2,70 & 2,98 & 6,86 & 3,94 & 3,81 \\
Cobre & 2,98 & 2,58 & 2,62 & 3,69 & 2,79 \\
Total & 23,67 & 18,60 & 20,31 & 23,71 & 24,13 \\
\hline
\end{tabular}


Foram verificadas pequenas variações nos teores de minerais encontrados nos rizomas avaliados.

Para o mineral sódio, o maior gradiente $(2,26$ vezes) foi verificado nas variedades cem/um $(9,26 \mathrm{ppm})$ e chinês $(4,09 \mathrm{ppm})$, enquanto que as variedades branco $(7,59 \mathrm{ppm})$, japonês $(7,91 \mathrm{ppm})$ e macaquinho $(8,96$ $\mathrm{ppm}$ ) apresentaram valores próximos entre si.

Os teores de ferro mostraram-se mais elevados nos inhames cem/um $(5,41$ ppm) e macaquinho (5,18 ppm), sendo que a variedade branco apresentou menor concentração deste mineral (3,87 ppm).

Quanto aos minerais manganês, zinco e cobre, os valores encontrados foram próximos (2,58 a 3,96 ppm, 2,70 a 3,94 ppm e 2,58 a 3,69 ppm, respectivamente) para todas as variedades estudadas, exceto a variedade chinês com 6,86 ppm de zinco e a branco com 1,58 ppm de manganês.

O total de minerais micro nutrientes coloca a variedade macaquinho como a de maior concentração $(24,13 \mathrm{ppm})$ e a variedade branco como a de menor (18,60 ppm).

\section{CONCLUSÃO}

Os resultados obtidos permitem concluir que:

- O inhame macaquinho apresentou maior porcentagem de umidade, seguido em ordem decrescente pelas variedades japonês, cem/um, branco e chinês.

- As variedades chinês e japonês apresentaram teores de proteínas equivalentes e superiores aos dos demais cultivares.

- Todos os inhames mostraram baixo conteúdo de lipídios.

- As variedades estudadas apresentaram diferentes valores de fibra, variando de 1,31 a 2,93\% $\mathrm{p} / \mathrm{p}$.

- O inhame japonês apresentou teor de resíduo mineral fixo superior $(1,15 \% \mathrm{p} / \mathrm{p})$ ao das outras variedades.

- A variedade chinês apresentou teor de amido significativamente superior, constituindo-se na melhor fonte de energia.

- Os inhames japonês e macaquinho foram as melhores fontes de minerais macro nutrientes e micro nutrientes, respectivamente. 
- A variedade chinês mostrou-se a matéria-prima de melhor aproveitamento industrial.

\begin{abstract}
Taro tuber root samples (Colocasia esculenta L. Schott) were analyzed aiming to establish and evaluate results of chemical composition and mineral nutrient contents, among different varieties (cem/um, branco, chinês, japonês and macaquinho). The studied varieties were experimentally cultivated in "Base de Estudos do Pantanal", UFMS, Brazil. The moisture content ranged from 62.78 (chinês) to $78.92 \% \mathrm{w} / \mathrm{w}$ (macaquinho). The chinês and the japonês varieties showed equivalent protein content $(2.86$ and $3.02 \% \mathrm{w} / \mathrm{w}$, respectively) and these values were higher than others were. All samples indicated poor lipid content $(0.21$ to $0.23 \% \mathrm{w} / \mathrm{w})$. In relation to the fiber fraction, the results differed among the varieties, from 1.31 to $2.93 \% \mathrm{w} / \mathrm{w}$. The japonês samples presented the highest ash content $(1.15 \% \mathrm{w} / \mathrm{w})$ indicating a rich content in macronutrient minerals $(218.35 \mathrm{mg} / 100 \mathrm{~g}$ of sample). The variety macaquinho presented the highest content of micronutrient minerals $(24.13 \mathrm{ppm})$. The concentration of starch found in the analyzed cultivars ranged from 14.46 to $30.30 \% \mathrm{w} / \mathrm{w}$, finding the highest value in the chinês variety. The evaluation of results of the handled analytical determinations showed up that the variety chinês is the best energy source, indicating that this cultivar could have an interesting industrial profitability, in relation to the other varieties.
\end{abstract}

\title{
REFERÊNCIAS BIBLIOGRÁFICAS
}

\section{ASSOCIATION OF OFFICIAL ANALYTICAL CHEMISTS. Official methods of analysis of the Association of Official Analytical Chemists. 14. ed. Washington, D.C., 1984. p. 988.}

2 AUED, S., CARVALHO, J.B., TAVARES, M., ZANELATTO, A.M. \& BACETTI,L.B. Determinação de amido em salsichas: comparação entre os métodos de Fehling e de Somogyi-Nelson e avaliação de metodologia para extração do amido. R. Inst. Adolfo Lutz, São Paulo, v. 50, n. 1/2, p. 251-6, 1989.

3 CHAN Jr., H.T., KAO-JAO, T.H. Anthocyanin composition of taro. J. Food Sci., Chicago, v. 42, p. 19-21, 1977.

4 CORREAA, M.P. Dicionário das plantas úteis do Brasil e das exóticas cultivadas. Rio de Janeiro : Instituto Brasileiro de Desenvolvimento Florestal, 1984. v. 4, p. 307-8.

5 FILGUEIRA, F.A.R. Manual de olericultura: cultura e comercialização de hortaliças. 2. ed. São Paulo : Agronômica Ceres, 1981. p. 297-302. 
6 GOERING, K.J., DeHAAS, D. New starches. VIII.Properties of small granules-starches from Colocasia esculenta. Cereal Chemistry, Minnesota, v. 49, n. 6, p. 712-9, nov./dec., 1972.

7 GOMES, F.P. Curso de estatística experimental. 10. ed. Piracicaba : Nobel, 1982. 468 p.

8 HEREDIA, M.C.V., BURBA, J.L., CASALI, V.M.D. In: SEMINÁRIO DE OLERICULTURA, 6., Viçosa, 1983. Anais... Viçosa:

Universidade Federal de Viçosa, 1983. 180 p.

9 INSTITUTO ADOLFO LUTZ. Normas analíticas do Instituto Adolfo Lutz: métodos químicos e físicos para análise de alimentos. 3. ed. São Paulo, 1985 . v. 1, p. 21-2, 27, 42-3, 53-4.

10 MENDEZ, M.H.M. et al. Método da fibra detergente neutro modificado para amostras ricas em amido. Ciênc. Tecnol. Aliment., Niterói, v. 5 , n. 2, p. 123-31, 1985.

11 MOY, J.H., NIP, W.K., LAI, A.O., TSAI, W.Y.J., NAKAYAMA,T.O.M. Development of extruded taro products. J. Food Sci., Chicago, v. 45, p. 652-6, 1980.

12 , SHADBOLT, B., STOENSAND, G.S., NAKAYAMA, T.O.M. The acridity factor in taro processing. J. Food Proc. and Preserv., Connecticut, v. 3, p. 139-44, 1987.

13 NATIONAL ACADEMY OF SCIENCES. Taro-roots and tubers. In: UNDEREXPLOITED tropical plants with promissing economic value. Washington, D.C., 1975. p. 37-43.

14 OPUTE, F.I., OSAGIE, A.U. Fatty acids composition of total lipids from some tropical storage organs. J. Sci. Food Agric., v. 29, p. 95962, 1978.

15 PIMENTEL, A.A.M.P. Olericultura no trópico úmido: hortaliças na amazônia. São Paulo : Agronômica Ceres, 1972. p. 245-51.

16 SALINAS, Y.G., GARCIA, R. Métodos químicos para el analises de suelos ácidos y plantas forrajeras. Colômbia, Cali : Centro Internacional de Agricultura Tropical, 1985. 83 p.

17 SIVIERO, M.L., FERREIRA, V.L.P., VITTI, P. \& SILVEIRA,E.T.F. Processamento e uso de farinha de inhame (Colocasia esculenta L. Schott) em produtos de panificação. Boletim do ITAL, Campinas, v. 21, n. 3, p. 355-80, 1984.

18 VAN SOEST, P.J. Use of detergent in the analysis of fibrous feeds. I. Preparation of fiber residues of low nitrogen content. J. Ass. 
Agric. Chem., Washington, D.C., v. 46, p. 825-9, 1963.

19 ZARATE, N.A.H., CASALI, V.W.D., ALVAREZ V,V.H. \& SILVA, J.F.

Curvas de crescimento vegetativo do inhame (Colocasia esculenta L. Schott), "chinês" e "macaquinho", considerando cinco populações, cultivados em solo seco. R. Cient. Cult., Campo Grande, v. 3, n. 1, p. 28-35, 1988. 